\title{
Value of MRUrethrographyin Comparison to Conventional RetrogradeUrethrography in the Surgical Treatment of Occlusive AnteriorUrethral Stricture
}

\author{
Dr.V.Sudhakar MD,RD ${ }^{1}$, Dr. S.Senthil Kumar DM,RD ${ }^{2}$, \\ ${ }^{1}$ Senior Assistant Professor OfRadiology, Government Kilpauk Medical College, Chennai. \\ ${ }^{2}$ Senior Resident OfRadiology, Government Kilpauk Medical College, Chennai.
}

\begin{abstract}
:
Objective: To prospectively assess the value of MR urethrography compared to conventional Retrograde urethrography in the surgical management of obliterative anterior urethral stricture.

Materials And Methods: 15 male patients along with 2 controls in the age group of 20-65, who presented to the radiology department with symptoms of urinary retention and in whom conventional retrograde urethrography $(R U G)$ had been performed were taken up for study.MR urethrography (MRU)was performed in these patients after a time interval of about 1 week, were analysed and it was focused on location, number, length and the signal intensity of the stricture.Anterior urethral stricture is measured along the long axis of the fibrotic segment shown as low signal intensity on the sagittal T2-weighted images. Stricture length on RUG was determined by measuring the distance between the proximal end of the distal distended urethra and the distal end of the open proximal urethra.Strictures with length of $<2.5$ were defined as "short strictures", where as those $>2.5$ as "long strictures". Additionally, MR findings were evaluated with regard to the urethra proximal to the stricture, the corpora spongiosa surrounding the stricture, adjacent organ injuries, and the associated complications like fistulas, sinus tracts etc.,

Results And Analysis: Paired T test correlations was performed to correlate the imaging and surgical length of stricture. The mean measurement errors between the imaging and surgical values of stricture length were calculated in each imaging method.MRU proved to be 100\% sensitive and specific comparedto RUG with a sensitivity and specificity of $80 \%, 33 \%$ for anteriorurethral stricture.

Conclusion: MR Urethrography using T2 weighted sequences can be a valuable means of imaging patients with anterior urethral strictures as well as rest of urinary system.From our study MRU depicted stricture length, periurethral fibrosis and also associated pathologies with accuracy, there by assisting the surgeons to select the most appropriate surgical procedure.
\end{abstract}

Keywords:

MRUMR Urethrography

RUGRetrograde Urethrography

RARERapid acquisition with relaxation enhancement

HASTEHalf Fourier Acquisition of Single Shot Turbo Spin Echo sequences

Anterior urethral stricture

Sagittal T2 weighted image.

Periurethral fibrosis

Fistulas, sinus tracts etc.

Roof of the penile bulb

\section{Introduction}

Imaging of the urinary tract with radiographs and contrast media played important role in the diagnosis of disorders of urinary tract for decades. The first ever retrograde pyelogram was done by VoelckerF,Von Lichtenberg (1). Intravenous urography gives a good anatomic detail of the urinary system and provides semi quantitative details about the renal function. Retrogradeurethrographyis considered the investigation of choice to image urethra

\subsection{Retrograde urethrogram}

The technique was first found by Cunningham in 1910 (2).It is a simple and cost effective investigation. It ruled over centuries in the evaluation of urethral pathologies. Fluoroscopy unit is a must.Nash PA, McAninch J (4) studied the anterior urethral strictures by RUG and showed that, there was good correlation of penile urethral stricture when compared to bulbar urethral stricture. 
This needs no patient preparation except for the local area cleanliness. About $20 \mathrm{ml}$ of water soluable contrast either HOCM or LOCM can be used. Prewarming of the contrast helps to reduce urethral spasm. Coned supine PA view of bladder base and urethra is taken. With aseptic precautions penile clamp is applied or tip of the balloon catheter placed in fossa navicularis and its balloon is filled with1-2ml water.

Contrast medium is infused in to the anterior urethra under fluoroscopic guidance and films are taken in the following positions.

1. Supine PA

2. 30 degree LAO, with leg abducted and knee flexed

3. 30 degree RAO, with left leg abducted and knee flexed

\section{Image analysis}

Full length of urethra is viewed for narrowing, mucosal irregularities, any fistulas sinus tracts.

The stricture length is measured between the tapered ends.

\section{Limitations of study}

The length of the stricture involving bulbar urethra is slightly under estimated by this modality. It also causes $0.5-1.6 \%$ of infections. There is risk of allergic reactions due to contrast, radiation exposure to testis (5-9 $\mathrm{msv}$ ), equal 230 chest radiographs. It does not give information about the extent of periurethral fibrosis. Intravasation of contrast can occur if excessive pressure is given to when injecting the contrast to overcome a stricture. Also urethral spasm may sometime occur which hinders the assessment of urethral pathology.

\subsection{MR Urethrography}

In imaging of the urethra Magnetic resonance imaging (MRI)is an excellent alternative tool. It is possible to rapidly acquire images of the urethra which are more informative than RUG even without the administration of contrast media with the RARE (Rapid acquisition with relaxation enhancement) and HASTE (Half Fourier Acquisition of Single Shot Turbo Spin Echo) sequences. This can overcome many of the drawbacks associated with RUG (3). With few of its drawbacks, MRU $(5,6)$, can be done to study the abnormalities of the urethra in a variety of situations, successfully. This study is an attempt to study the diagnostic capability and efficacy of MRU, in the visualization of anterior urethra in comparison to RUG andmost importantlyits value in providing the information required by the surgeon to plan the most appropriate surgical procedure.

\section{Materials And Methods}

15 male patients along with 2 controls in the age group of 20-65, who presented to the radiology department with symptoms of urinary retention and in whom RUG had been performed were taken up for study. The study was performed in SIEMENS MAGNETOM Symphony 1.5 Tesla MRI scanner available at our institute.HASTE sequence was obtained in each patient using a torso pelvic phased array coil, as described below.

Period of study: 3 Years

\subsection{Inclusion Criteria}

Cases

15 male patients in the age group of 20-65 years with history of straddle injury, transurethral instrument manipulation or urethritis presenting with symptoms of urethral stricture, in those patients RUGhas been done for suspected urethral stricture.

After a time interval of about 1 week,MRU was performed in these patients.

\section{Controls}

2 male patients who underwent screening for symptoms of urethral stricture for whom MRU is normal.

\subsection{Exclusion Criteria}

Patients with metallic implants like cardiacpacemaker, implanted cardiac defibrillator, cochlear implants etc., severe hypersensitivity or previous allergic reactions, claustrophobia andcritically ill patients. 


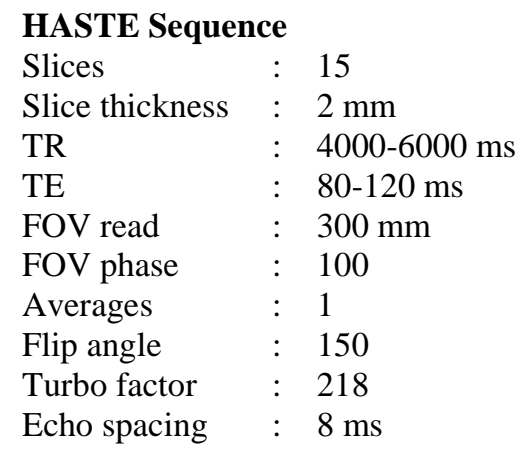

Patient preparation: No specific preparation.

Patient position: Head first and supine.

\subsection{Procedure}

\section{MR Urethrography}

Patient is placed in Torso pelvic phased array coil. The tip of a $10 \mathrm{ml}$ syringe filled with $8-10 \mathrm{ml}$ of sterile lubricating gel was inserted into the external urethral meatus. The sterile lubricating jelly was infused in to the anterior urethra until resistance was felt and some of the lubricating jelly overflowed from the urethral meatus. The glans sulcus of the penis was then gently tied by using long gauze in order to avoid escape of the lubricating jelly. The penile shaft was secured in the midsagittal position of the pelvis by using an upward traction and taping the edges of the tying gauze to the abdomen.High resolution sagittal T2 weighted images of penis and bladder was acquired. The center of the localizer was placed over the symphysis to cover the bladder and urethra and images were obtained.

\subsection{Image Analysis}

MRU

Obtained images were analysed and it was focused on location, number, length and the signal intensity of the stricture in MRU.Anterior urethral stricture is measured along the long axis of the fibrotic segment shown as low signal intensity on the sagittal T2-weighted images.Extent of periurethral fibrosis was assessed by characterizing the surrounding depth and hypointensity of periurethral tissue.

Additionally, MR findings were evaluated with regard to the urethra proximal to the stricture, the corpora spongiosa surrounding the stricture, adjacent organ injuries, and the associated complications like fistulas, sinus tracts etc.,

\subsection{Conventional retrograde urethrography (RUG)}

Stricture length on RUG was determined by measuring the distance between the proximal end of the distal distended urethra and the distal end of the open proximal urethra.

Strictures with length of $<2.5$ were defined as "short strictures", where as those $>2.5$ as "long strictures".

3.1. Case. 1

\section{Representative Images}
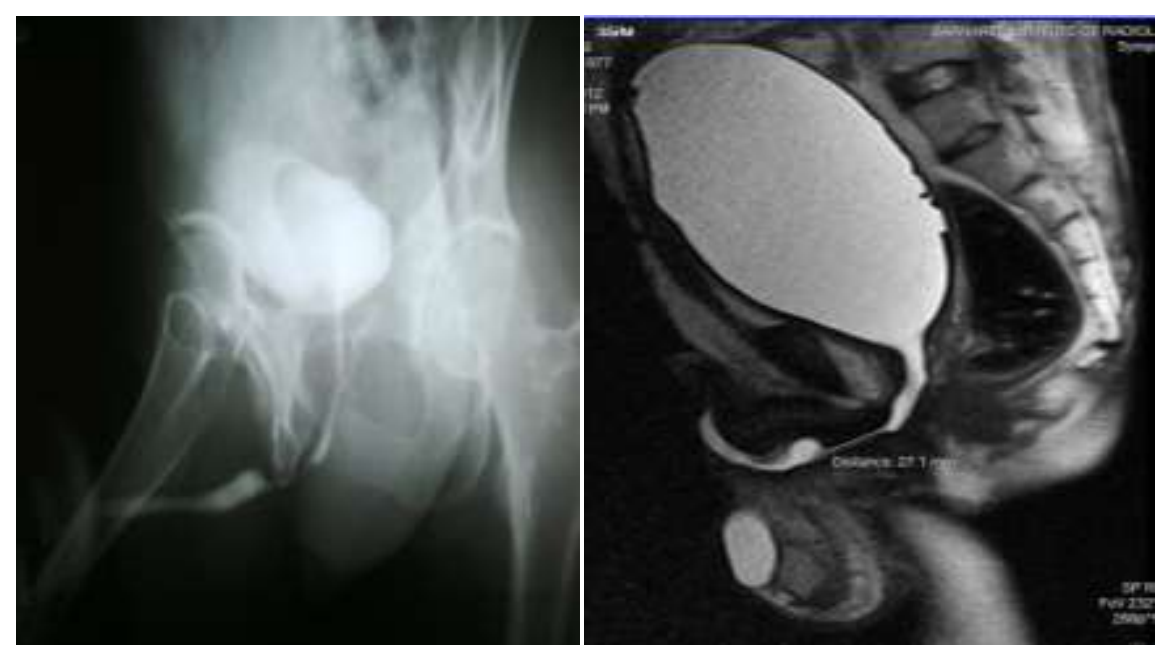
35 years old patient with history of straddle injury. RUG underestimated the length of anterior urethral stricture as determined by the distance between the distance between the proximal end of the distal distended urethra and the distal end of the open proximal urethra. Whereas MRU depicted the accurate stricture length as shown by hypointense signal between the proximal and distal ends of the dilated urethra as well as the extent of peri urethral fibrosis on the sagittal T2-weighted HASTE images.

\subsection{CASE.2}

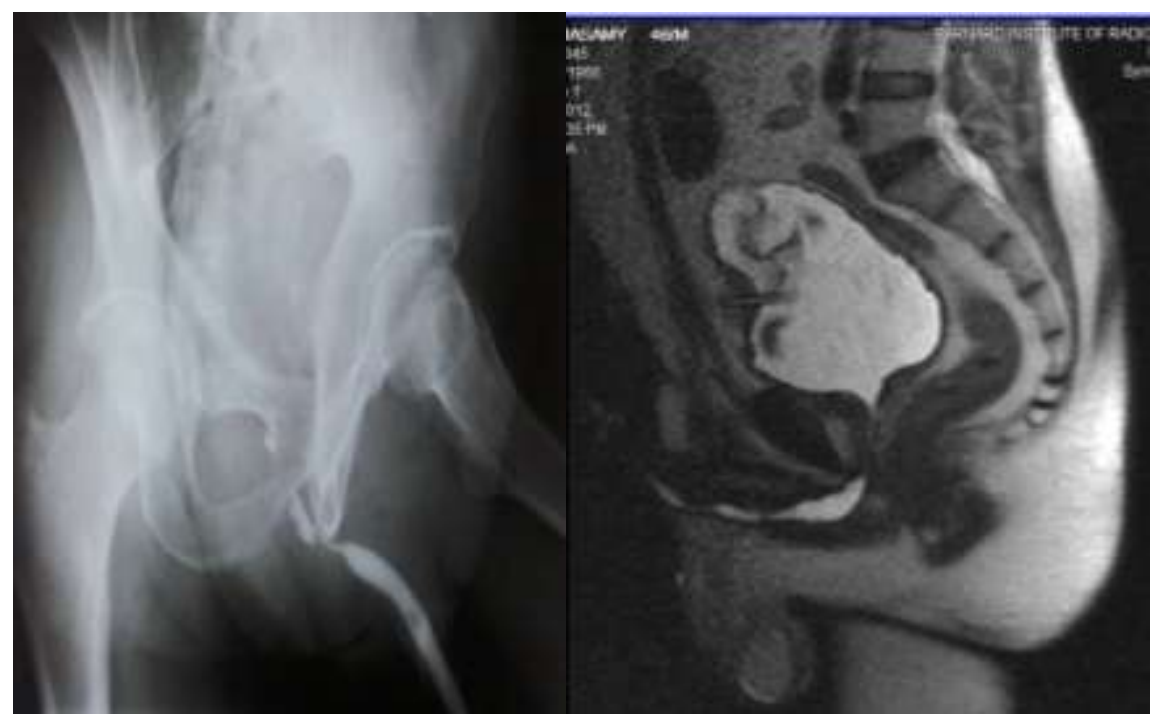

33years old patient with history of recurrent urethritis. RUG shows single short segment bulbar urethral stricture. MRU showed multiple penile and bulbar urethral strictures of varying length with mucosal irregularities on sagittal HASTE images.

\section{Results}

Initially planned the surgical procedure according to conventional retrograde urethrography findings, then changed, based on MRU findings. The results of each imaging method were compared with a description of the surgical findings.

\subsection{Surgical Procedures}

Endoscopic internal urethrotomy $-0.5-1.5 \mathrm{~cm}$.

Primary anastomotic urethroplasty up to $2.5 \mathrm{~cm}$.

Complex urethroplasty- more than $2.5 \mathrm{~cm}$.

\subsection{Statistical Analysis}

Table.1 Group Statistics

\begin{tabular}{|l|l|l|l|l|l|}
\hline & Stricture & N & Mean & Std. Deviation & Std. Error Mean \\
\hline Conventional RUG - Length & Anterior & 15 & 1.700 & 1.2553 & .3241 \\
\hline MR Urethrography - Length & Anterior & 15 & 2.033 & 1.1312 & .2921 \\
\hline Surgical length & Anterior & 15 & 2.040 & 1.2477 & .3222 \\
\hline
\end{tabular}

Paired $\mathrm{T}$ test correlations was performed to correlate the imaging and surgical length of stricture. Thestandard mean measurement errors and standard deviation between the imaging and surgical values of stricture length were calculated in each imaging method.

Table.2T-Test - Anterior urethral strictures -Paired Samples Statistics

\begin{tabular}{|l|l|l|l|l|l|}
\hline \multicolumn{2}{|l|}{} & Mean & N & Std. Deviation & Std. Error Mean \\
\hline Pair 1 & Conventional RUG - Length & 1.700 & 15 & 1.2553 & .3241 \\
\hline & MR Urethrography - Length & 2.033 & 15 & 1.1312 & .2921 \\
\hline Pair 2 & Conventional RUG - Length & 1.700 & 15 & 1.2553 & .3241 \\
\hline & Surgical length & 2.040 & 15 & 1.2477 & .3222 \\
\hline Pair 3 & MR Urethrography - Length & 2.033 & 15 & 1.1312 & .2921 \\
\hline & Surgical length & 2.040 & 15 & 1.2477 & .3222 \\
\hline
\end{tabular}


Value Of Mrurethrography In Comparison To Conventional Retrogradeurethrography In The...

Table.3 Paired Samples Correlations

\begin{tabular}{|l|l|l|l|c|}
\hline \multicolumn{2}{|l|}{ Conventional RUG - Length \&MRUrethrography - Length } & N & Correlation & Sig. \\
\hline Pair 1 & C & .887 & .001 \\
\hline Pair 2 & Conventional RUG - Length \& Surgical length & 15 & .898 & .001 \\
\hline Pair 3 & MR Urethrography - Length \& Surgical length & 15 & .986 & .001 \\
\hline
\end{tabular}

A $\mathrm{P}$ value $<.05$ is considered to indicate a significant difference.

\section{Discussion}

Strictures with length of $<2.5 \mathrm{~cm}$ were defined as "short strictures", whereas $>2.5 \mathrm{~cm}$ as "long strictures".There are many treatment options for obliterative urethral strictures. The optionschosen depends on several factors, most important being the stricture length(7). Endoscopic treatment can be useful for a thin septum like stricture $0.5-1.5 \mathrm{~cm}(8)$. A short urethral stricture measuring $<2.5 \mathrm{~cm}$, is amenable to be managed with primary anastomotic urethroplasty (9). Whereas a complex technique involving a graft or flap is generally performed in patients with a long urethral strictures measuring $>2.5 \mathrm{~cm}$. (10)Primary anastomotic urethroplasty is mainly done by the perineal route. (11)

The other factors that determine the choice of repair include the stricture causes, the extent of fibrosis of the corpora spongiosa, prior surgical treatment and the surgeon's option. Therefore a careful evaluation of the obliterative urethral stricture is most important in the surgical management (12). RUG is the method of choice among investigative procedures for the planning of a urethral reconstruction.

As for as the anterior urethral stricture, its length is underestimated if the patient is not properly positioned due to normal anatomical fixity of the bulbar urethra along the plane of axis of the pelvis leading to "end on view". It requires a steep oblique position for exact localisation while performing RGU $(13,14)$

\section{Conclusion}

RUG is well known established technique in delineating anterior urethral strictures. Although readily available and cost effective do not determine the accurate length andperiurethral fibrosis. The anterior urethral stricture may be underestimated due to problems of positioning. It also requires need for administration of potentially risk contrast media, the relatively significant amount of radiation exposure especially in younger patients.

MRU can be a valuable means of imaging patients with urethral strictures. T2 weighted sequences are excellent for demonstrating urethra as well as rest of urinary system. MR imaging is especially useful in planning the surgical procedure in cases of anterior urethral strictures and the extent of periurethral fibrosis. It accurately shows the stricture length and dictates the surgeon to plan the surgery via the perineal (or) transpubic approach. In this condition only strictures upto $1.5 \mathrm{~cm}$ are amenable for endoscopic urethrotomy. Even with these short strictures with extensive periurethral fibrosis, dilatations and endoscopic urethrotomy could not be done and requires primary anastomotic urethroplasty.

MRU can be successfully performed in patients in whom contrast studies are contraindicated. Paediatric population can also be subjected to MRU without radiation to testis.From our study, the stricture length,periurethral fibrosis and also associated pathologies are accurately determined by MRU thereby helping surgeons to the select most appropriate surgical procedure.

\section{Acknowledgements}

Prof.J.DeviMeenal MD, DNB, Professor, Kilpauk Medical College,Chennai.

Prof.S.Babu Peter MD,DNB, Professor, Barnard institute of Radiology,Madras Medical College,Chennai.

Prof.C.Amarnath MD,DNB, Professor, Stanley Medical College,Chennai.

Prof.N.KailasanathanMD,DMRD, Professor and Director, Barnard institute of Radiology, Madras Medical College, Chennai.

\section{References}

[1]. Tuffier T. Sondeureterale opaque. In: Duplay SE, Reclus P, eds. Traite' de chirurgie. Paris: Masson, 1897; 412-413

[2]. Cunninham J. The diagnosis of stricture of the urethra by Roentgenrays. Trans Am AssocGenitourinSurg 1910;5:369-371.

[3]. Y Osman - European Urology, MR Urethrography in Comparison to Retrograde Urethrography in Diagnosis of Male urethral strictures 50/3,587-594, September 2006.

[4]. Nash PA, McAninch JW, Bruce JE, Hanks DK. Sono-urethrography in the evaluation of anterior urethral strictures J Urol. 1995 Jul;154(1):72-6.

[5]. Dixon CM, McAninch JW. MR imaging of urethral defects and pelvic crush injuries. Urol 1992;148:1162-5.

[6]. Narumi Y, Hricak H,.MR imaging of traumatic urethral injury. injury.radiology.rsna.org/content/188/2/439.

[7]. Peterson AC Webster GD.Management of urethral stricture disease: developing options for surgical intervention. BJU Int 2004;94:971-976.

[8]. Jordan GH, Schlossberg SM. Surgery of the penis and urethra. In: Walsh PC, Retik AB, Vaughan ED Jr, Wein AJ, eds. Campbell's urology. 8th ed. Philadelphia, Pa: Saunders, 2002; 3886-3952.

[9]. Santucci RA, Mario LA, McAninch JW. Anastomotic urethroplasty for bulbar urethral stricture: analysis of 168 patients. J Urol 2002;167:1715-1719. 
Value Of Mrurethrography In Comparison To Conventional Retrogradeurethrography In The...

[10]. Goel MC, Kumar M, Kapoor R. Endoscopic management of traumatic posterior urethral stricture: early results and followup. J Urol 1997; $157: 95$

[11]. Webster GD, Ramon J. Repair of pelvic fracture posterior urethral defects using an elaborated perineal approach: experience with 74 cases. J Urol 1991;145: 744-748.

[12]. MacDonald MF, Santucci RA. Review and treatment algorithm of open surgical techniques for management of urethral strictures. Urology 2005;65:9-15.

[13]. Kawashima A, Sandler CM, Wasserman NF, LeRoy AJ, King BF Jr, Goldman SM. Imaging of urethral disease: a pictorial review. RadioGraphics 2004; 24(Spec Issue):S195-S216.

[14]. Syed Maтun Mahmud, Salman El Khalid, Abdul MajidRana, ZafarZaidi.Isascending urethrogrammandatory for all Urethral Strictures? Jan 2001 to Dec 2002 